\title{
Equipamento Vestível para Auxílio na Mobilidade de Pessoas Com Deficiência Visual
}

\author{
Luis Claudio Leite Pereira \\ Universidade do Estado de Santa Catarina \\ Joinville, Santa Catarina \\ luiscla1987@gmail.com
}

\author{
Janine Kniess \\ Universidade do Estado de Santa Catarina \\ Joinville, Santa Catarina \\ janine.kniess@udesc.br
}

\begin{abstract}
Mobility for people with visual impairments is a challenge in places where there is no knowledge of obstacles. Research carried out in this work identified that people with visual impairment have difficulties with obstacles located above 1 meter. Thus, an approach is proposed to notify the visually impaired person through sound and vibration about such obstacles. The proposed solution is available on the ThingSpeak platform and components such as microcontrollers (ESP8266 NodeMcu ESP-12), sensors, buzzer and GPS were used in its development. Results confirmed that the proposed approach correctly identified the existence of obstacles with a height equal to or greater than 1 (one) meter in the way of visually impaired people.
\end{abstract}

\section{KEYWORDS}

Equipamento Vestível, Deficiência Visual, Mobilidade

\section{INTRODUÇÃO}

No Brasil existem cerca de 45,6 milhões de pessoas que declaram ter pelo menos um tipo de deficiência, ou seja, 23,9\% da população [3]. O Sul é a região com maior proporção de pessoas com deficiência visual, 5,4\% da população [3]. Pesquisas realizadas na Associação de Deficientes Visuais da cidade de Mafra-SC indicaram que pessoas com deficiência visual tem dificuldade com obstáculos altos, principalmente lixeiras basculantes instaladas em muros e que obstruem as calçadas. A pesquisa foi realizada in loco e sete pessoas com deficiência visual participaram. Os entrevistados relataram que as principais dificuldades são: mobilidade onde há obstáculos altos e buracos no caminho.

Neste contexto, o desenvolvimento de soluções para auxiliar pessoas com deficiência visual a identificarem obstáculos altos, com altura igual ou superior a 1 metro de altura, ainda é uma questão em aberto. Com este objetivo, propõe-se neste trabalho um equipamento vestível que auxilia na identificação de obstáculos altos.

Outra contribuição apresentada neste trabalho é o desenvolvimento de uma rede social para que os obstáculos altos possam ser cadastrados pela comunidade de interesse, visando alertar o deficiente visual em relação aos obstáculos já cadastrados.

Na rede social, a posição geográfica dos obstáculos altos pode ser previamente cadastrada via interface web (ver Figura 3). Um aplicativo Android também foi desenvolvido para o cadastro prévio dos obstáculos na rede social. Durante o percurso, o utilizador do equipamento vestível é notificado sobre a existência de obstáculos já cadastrados no sistema. Além disso, o equipamento vestível, através de sensores, notifica o utilizador sobre a existência de novos obstáculos no caminho.
Um sensor ultrassônico acoplado ao protótipo identifica os obstáculos altos, com altura igual ou superior a 1 metro e a uma distância inferior a 130 centímetros. Definiu-se a distância entre o obstáculo e o protótipo baseado no tamanho médio das bengalas usadas pelas PCDV, 110 centímetros.

Para notificar a pessoa com PCDV sobre a existência dos obstáculos já cadastrados na base de dados e que estão no percurso do utilizador do equipamento vestível, desenvolveu-se uma pulseira com um micro vibrador motor que por meio de beeps informa a presença do obstáculo (ver Tabela 2).

Este trabalho está organizado como segue: são apresentados na Seção 2 os trabalhos relacionados. Na Seção 3, o protótipo do equipamento vestível e o funcionamento da rede social são descritos. Na Seção 4, os resultados são apresentados. Por fim, na Seção 5, as conclusões e trabalhos futuros.

\section{TRABALHOS RELACIONADOS}

Nesta seção, discute-se os trabalhos relacionados com a abordagem proposta neste artigo. No trabalho de [7], denominado IoT based Walking Cane for Typhlotic with Voice Assistance, propõe-se o desenvolvimento de uma bengala inteligente, para substituir a bengala convencional. A bengala possui cinco sensores ultrassônicos que identificam obstáculos no caminho. Dentre os sensores, quatro são utilizados para verificar obstáculos à frente, o outro sensor é responsável por verificar a existência de buracos. O usuário é informado sobre os obstáculos por meio de um buzzer que emite alarmes. O sistema também converte texto em fala. Frases predefinidas são convertidas em fala, por exemplo: "Staircase ahead", o utilizador é informado por um fone. A abordagem contempla rastreamento em tempo real, porém não informa da existência de obstáculos com altura superior a 1 metro.

O protótipo de [5] consiste em uma bengala inteligente para substituir a bengala convencional, composto por: 1 Arduíno uno, 1 sensor ultrassônico e 1 buzzer. A abordagem proposta faz a leitura de obstáculos, e avisa sonoramente o usuário com alarmes. Quanto mais próximo do obstáculo, mais curto o intervalo dos alarmes.

O protótipo, Auxílio de Deficientes Visuais (PADEVI) de [2] utiliza sensores do Kinect para coletar informações do ambiente que são manipuladas por microcontroladores. As informações são passadas ao utilizador por meio de um fone de ouvido. O protótipo visa auxiliar nas limitações da bengala tradicional: avisar sobre obstáculos altos como, telefones públicos e informar sobre buracos no percurso. Com o Kinect, o protótipo consegue diferenciar obstáculos de pessoas durante o percurso do utilizador. Contudo, o uso do Kinect aumenta o custo de desenvolvimento do projeto.

No trabalho de [4], Navigation Aidinng Stick for the Visually Impaired, apresenta-se uma bengala que verifica a existência de 
obstáculos no percurso do utilizador para substituir a bengala convencional. O protótipo é composto por: 1 Arduíno Mega 2560, 4 sensores ultrassônicos, 1 módulo GPS, 1 dispositivo de áudio e 1 motor vibratório. Os sensores ultrassônicos são dispostos na bengala com o objetivo de fazer a leitura de obstáculos frontais e laterais. O dispositivo de áudio é programado para reproduzir frases pré cadastradas, por exemplo: "Turn Around" ou "Move Left", e a bengala sugere o caminho de acordo com a leitura dos sensores ultrassônicos. A bengala vibra para informar o utilizador da existência de um obstáculo em seu caminho. A abordagem não contempla a identificação de obstáculos com altura igual ou superior a 1 metro.

[6] propõe a ferramenta, BlinDar: An Invisible Eye for the Blind People. O BlinDar é composto por: 1 Arduíno Mega 2560, 3 sensores ultrassônicos, 1 sensor de gás (MQ2 GAS) para detecção de fogo ou fumaça, 1 módulo GPS, 1 buzzer, 1 módulo conversor de texto em voz e 1 motor vibratório. Os sensores ultrassônicos fazem a leitura da distância do usuário em relação aos obstáculos. Um módulo conversor de texto em fala é pré-programado com frases prontas para informar o utilizador da presença de obstáculos. O protótipo verifica posição geográfica do utilizador e a disponibiliza na plataforma ThingSpeak. Por focar na bengala, a proposta não faz leitura de objetos com altura superior a 1 metro.

Com exceção do trabalho de [2] que utiliza um Kinect, os demais trabalhos não detectam objetos com altura igual ou superior a 1 metro. Na Tabela 1, apresenta-se uma comparação entre os trabalhos relacionados discutidos nesta seção.

\begin{tabular}{|l|l|l|l|l|l|}
\hline Ref. & GPS & Rastro & $\begin{array}{l}\text { Obstáculo } \\
\geq 1 \mathrm{~m}\end{array}$ & $\begin{array}{l}\text { Rede } \\
\text { Social }\end{array}$ & Pulseira \\
\hline$[7]$ & SIM & SIM & NÃO & NÃO & NÃO \\
{$[5]$} & NÃO & NÃO & NÃO & NÃO & NÃO \\
{$[2]$} & NÃO & NÃO & SIM & NÃO & NÃO \\
{$[6]$} & SIM & SIM & NÃO & NÃO & NÃO \\
{$[4]$} & SIM & NÃO & NÃO & NÃO & NÃO \\
\hline
\end{tabular}

Table 1: Comparação dos Trabalhos Relacionados

A proposta apresentada nesse artigo se diferencia dos demais nos seguintes aspectos: (i) utilização de uma rede social que permite o registro prévio dos obstáculos. Por meio da rede social, pode-se avisar com antecedência o utilizador sobre a presença de obstáculos no caminho; (ii) leitura de objetos com altura igual ou superior a 1 metro. $\mathrm{O}$ equipamento vestível pode operar mesmo sem conexão com a Internet (nesse caso ocorre apenas a detecção de obstáculos com o sensor ultrassônico); (iii) uso de uma pulseira para informar através de beeps sobre os eventos que estão acontecendo, por exemplo, a existência de um obstáculo no caminho.

\section{EQUIPAMENTO VESTÍVEL}

O protótipo do equipamento vestível foi desenvolvido para colaborar com a bengala convencional porém, com o foco nas limitações da bengala em relação a identificação de objetos com altura igual ou superior a 1 metro, por exemplo, lixeiras basculantes e telefones públicos.

No desenvolvimento da abordagem proposta neste trabalho, foram adicionados 6 elementos conforme apresentado na Figura 1:
(1) Pulseira com micro vibrador motor; (2) Equipamento Vestível; (3) smartfone do utilizador; (4) Internet; (5) Banco de dados vinculado à rede social e; (6) Plataforma ThingSpeak.

O hardware do equipamento vestível (representado pelo número 2 na Figura 1) é transportado pelo utilizador, por exemplo, acoplado na alça de uma mochila (parte frontal). Na construção do equipamento, utilizou-se 1 ESP8266 NodeMcu ESP-12, 1 módulo GPS que tem precisão horizontal de até 2,5 metros, 1 sensor ultrassônico HC-SR04 e 1 buzzer. Os elementos que compõem o equipamento vestível são descritos na Seção 3.1.

O equipamento vestível, comunica-se com a Internet através de um smartfone que deve ser transportado pelo usuário. $\mathrm{O}$ microcontrolador do equipamento vestível, constantemente acessa o banco de dados de obstáculos cadastrados para receber a lista de obstáculos próximos da posição atual do usuário. O microcontrolador utilizado dispõe de um módulo para a conexão sem fio à Internet.

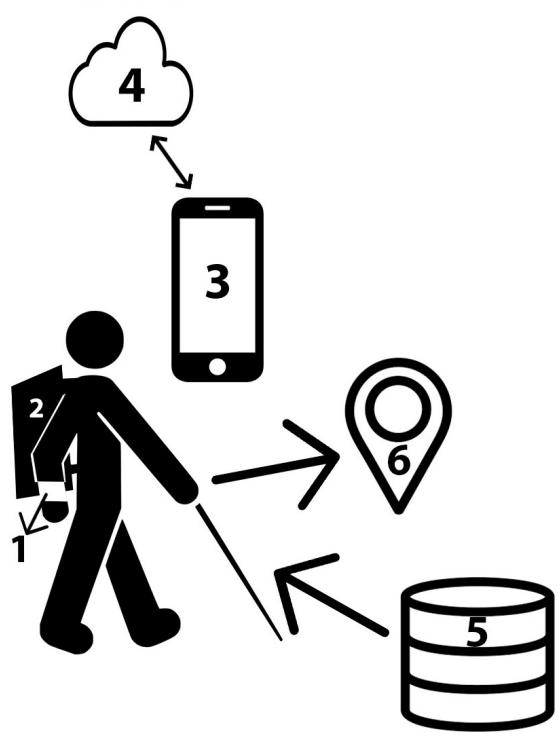

Figure 1: Elementos da Composição da Abordagem Proposta. Fonte: Próprio Autor.

A Figura 1 representa uma pessoa com deficiência visual caminhando. Em uma das mãos ela segura uma bengala e na outra carrega no pulso a pulseira com micro vibrador motor (representado pelo número 1 na Figura 1). Nas costas, a PCDV transporta uma mochila na qual o equipamento vestível (número 2) foi acoplado (em uma das alças na parte frontal da mochila). O smartfone é representado pelo número 3 e a Internet pelo número 4 . $\mathrm{O}$ banco de dados da rede social é representado pelo número 5 e a plataforma ThingSpeak pelo número 6.

O equipamento vestível (2) tem as seguintes responsabilidades: identificar obstáculos à frente através do sensor ultrassônico, verificar no banco de dados a existência de algum obstáculo previamente cadastrado, conectar o smartfone (3) do utilizador com a Internet e enviar informações sobre os obstáculos para a pulseira (1). No escopo deste trabalho, obstáculos identificados em tempo real durante 
o percurso do utilizador não são cadastrados automaticamente no sistema.

Por exemplo, o equipamento vestível verifica a existência de um obstáculo e envia um sinal para a pulseira. Então, a pulseira emite três beeps curtos (ver Tabela 2 para informar o utilizador da presença do obstáculo. Para essa função, o equipamento não precisa estar conectado à Internet.

O equipamento vestível utiliza o smartfone do utilizador para conectar-se a Internet (a conexão pode ser via rede celular ou WiFi). A escolha depende do módulo de conexão sem fio utilizado no microcontrolador. Após o estabelecimento da conexão com a Internet, o equipamento vestível analisa os obstáculos cadastrados no banco de dados, e com isso consegue informar com antecedência ao utilizador sobre a presença de obstáculos na região.

A pessoa com deficiência visual pode habilitar uma ou mais pessoas de seu interesse para receber um aviso sobre sua localização em caso de uma emergência ou quando desejar. Para obter a posição geográfica do utilizador, o microcontrolador utiliza a biblioteca TinyGPS [1], utilizando a latitude e longitude do utilizador como ponto de referência para o cálculo da localização.

\subsection{Características do Hardware}

No desenvolvimento da solução foi utilizado um módulo ESP8266 NodeMcu ESP-12 ${ }^{1}$, que faz o processamento, leitura e envio dos dados coletados, como por exemplo, a posição atual do utilizador do equipamento vestível. Os dados coletados e processados são armazenados na plataforma ThingSpeak.

Na pulseira, utilizou-se um ESP8266 ESP-01 que foi programado para receber o comando do equipamento vestível para acionar o motor vibratório e informar o usuário da existência dos obstáculos. O ESP8266 ESP-01 dispõe de um módulo Wifi para a comunicação com o equipamento vestível. O hardware adotado para obter a posição geográfica do utilizador é um módulo GPS NEO6M, com o protocolo NMEA [8]. O módulo GPS NEO6M tem baixo custo, e precisão de 2,5 metros horizontais.

Para reconhecer os obstáculos que estão na altura de 1 metro ou superior, utilizou-se sensores ultrassônico do modelo HC-SR $44^{2}$. O HC-SR04 tem um alcance de $2 \mathrm{~cm}$ a $4 \mathrm{~m}$ com um ângulo de leitura de $15^{\circ}$. O sensor é responsável por capturar a distância entre o objeto e o utilizador do equipamento vestível.

Após a captura da distância pelo sensor, a informação é transmitida ao módulo ESP8266 NodeMcu ESP-12. Nesse módulo são realizadas operações como: estabelecimento da conexão com a Internet, verificação de obstáculos e envio de dados ao utilizador.

Na Figura 2, demonstra-se o equipamento vestível visto de frente. O equipamento é composto pelos seguintes itens de hardware: um módulo GPS (representado pelo número 1 na Figura 2), um buzzer (2), um ESP8266 NodeMCU ESP-12 (3), um sensor ultrassônico (4) e uma fonte ajustável (5).

Os componentes, GPS, sensores, buzzer e conversor de tensão elétrica foram conectados no ESP8266 NodeMcu ESP-12 para obter a posição geográfica do utilizador e informar sobre a existência de

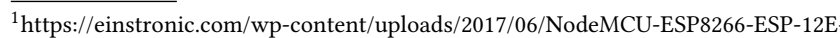
Catalogue.pdf

${ }^{2}$ https://www.mouser.com/ds/2/813/HCSR04-1022824.pdf
}

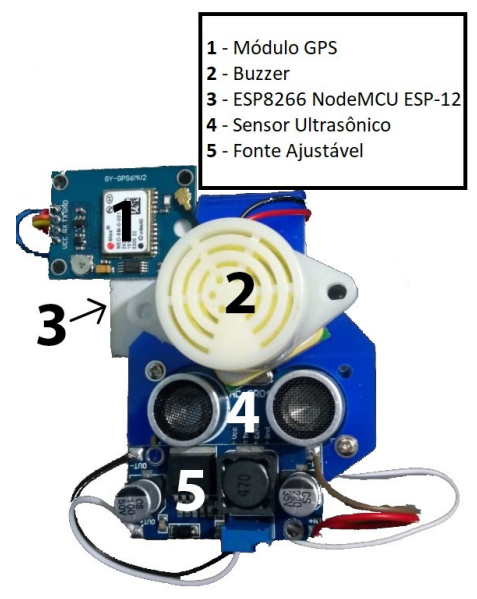

Figure 2: Componentes do Equipamento Vestível, Fonte: Próprio Autor.

obstáculos novos em seu percurso. Também, acoplou-se na pulseira, um módulo Wifi ESP8266 ESP-01 e um micro vibrador motor.

Através de vibrações emitidas pela pulseira (ver Tabela 2), o usuário pode identificar qual o tipo de evento está ocorrendo. Para a emissão dos beeps foi utilizado um buzzer. Os beeps e as vibrações acontecem simultaneamente.

\begin{tabular}{|l|l|}
\hline TIPO DO BEEP & Evento \\
\hline Um Beep Curto & $\begin{array}{l}\text { Ao ligar o protótipo a cada ten- } \\
\text { tativa de conexão com a rede, a } \\
\text { pulseira emitirá um beep curto. }\end{array}$ \\
\hline Três Beeps Curtos & $\begin{array}{l}\text { Obstáculo identificado a uma al- } \\
\text { tura igual ou superior a 1 metro e } \\
\text { a uma distância inferior a 130 cm. }\end{array}$ \\
\hline $\begin{array}{l}\text { Três Beeps Curtos e } \\
\text { um beep curto }\end{array}$ & $\begin{array}{l}\text { Após três beeps curtos haverá um } \\
\text { beep curto, se a informação de lo- } \\
\text { calização for salva com sucesso } \\
\text { no banco de dados. }\end{array}$ \\
\hline $\begin{array}{l}\text { Beep longo - 2 se- } \\
\text { gundos }\end{array}$ & $\begin{array}{l}\text { Informa que o protótipo conec- } \\
\text { tou com a Internet e está funcio- } \\
\text { nando. }\end{array}$ \\
\hline $\begin{array}{l}\text { Beep longo - 4 se- } \\
\text { gundos }\end{array}$ & $\begin{array}{l}\text { Informa que o GPS está realizando } \\
\text { a leitura da posição corretamente. }\end{array}$ \\
\hline Dois Beeps Curtos & $\begin{array}{l}\text { Dados foram inseridos na } \\
\text { plataforma ThingSpeak. }\end{array}$ \\
\hline
\end{tabular}

Table 2: Descrição dos Tipos de Eventos

O protótipo tem as seguintes dimensões: $9 \mathrm{~cm}$ de largura, $10 \mathrm{~cm}$ de altura e $8 \mathrm{~cm}$ de profundidade pesando aproximadamente 300 gramas. $\mathrm{O}$ equipamento pode ser fixado nas alças frontais de uma mochila. Como fonte de energia, sugere-se 4 pilhas do tipo AA, neste trabalho não foi medida a eficiência, e autonomia energética do protótipo. 


\subsection{Software Utilizado}

No aspecto lógico, o projeto é composto de três ferramentas: Rede Social (Figura 3), Aplicativo Android (Figura 4), e o software de funcionamento de protótipo.

A posição dos obstáculos é inserida previamente pelos interessados na rede social de duas maneiras: por um navegador da web, ou por um aplicativo Android. Em ambas, o usuário cadastra: latitude e longitude do obstáculo e o tipo.

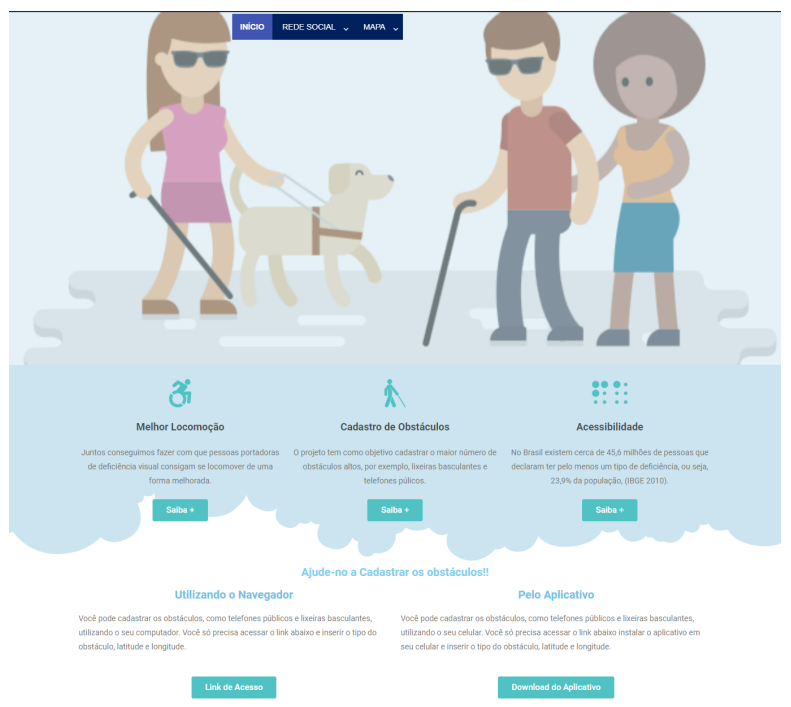

Figure 3: Interface da Rede Social. Fonte: Próprio Autor.

Na Figura 4, demonstra-se a interface do aplicativo Android. Na interface do Android, o usuário pode selecionar o tipo de obstáculo, ativar, desativar e enviar a localização através de três botões separadamente. Abaixo dos botões, tem-se um mapa mostrando a posição atual do obstáculo.

O software do equipamento vestível verifica a existência de obstáculos de dois modos: online e offline. No modo online, verifica-se na base de dados a presença de obstáculos previamente cadastrados em um raio de 50 metros em relação a posição atual do utilizador e obstáculos à frente do utilizador com distância inferior a 80 centímetros. No modo offline, faz-se somente leitura dos obstáculos à frente do usuário.

A posição em tempo real do utilizador é utilizada para avisá-lo da presença de obstáculos no caminho com base nos obstáculos já cadastrados no sistema. As informações de localização do utilizador são enviadas periodicamente para a plataforma ThingSpeak.

Os dados gerados através do protótipo são enviados para a plataforma ThingSpeak via protocolo da camada de aplicação MQTT (Message Queuing Telemetry Transport). O protocolo MQTT foi escolhido porque é um protocolo leve em comparação ao protocolo Hypertext Transfer Protocol (HTTP).

As informações do ThingSpeak podem ser acessadas via um sítio na web, ou através do App ThingView (Figura 5). No App ThingView o interessado insere as credenciais e pode visualizar em tempo real a posição geográfica da pessoa com deficiência visual. Esta última função foi adicionada ao projeto para permitir que a

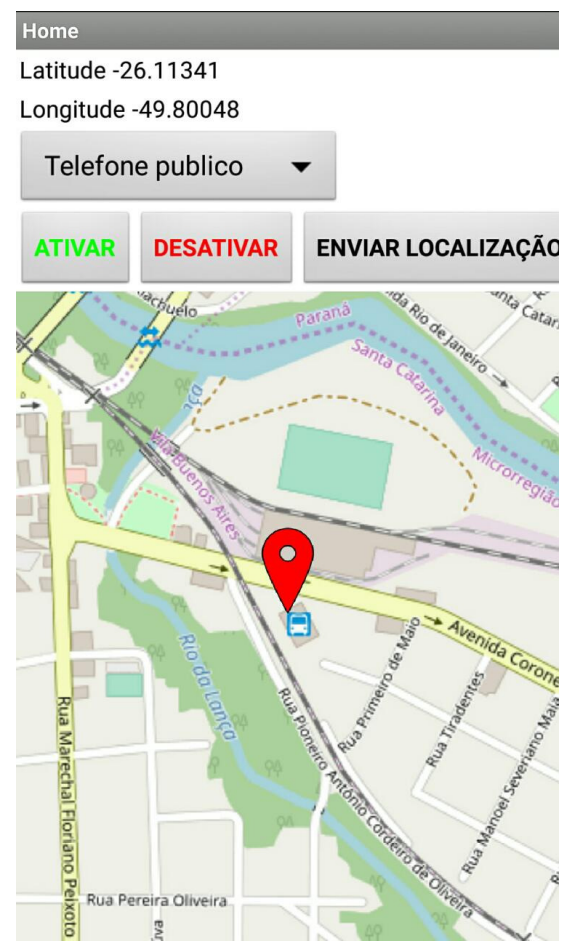

Figure 4: Interface do Aplicativo Android. Fonte: Próprio Autor.

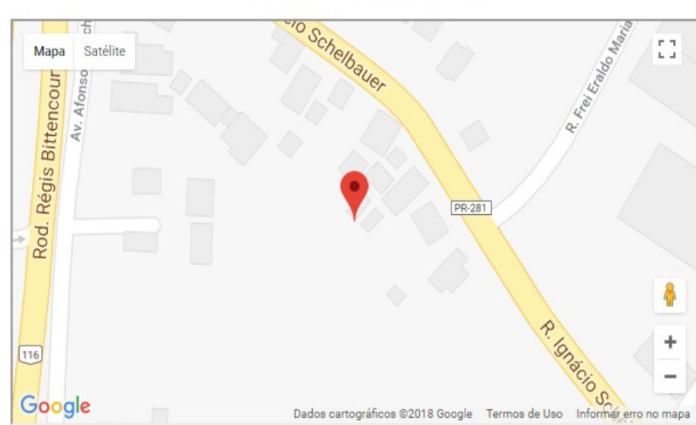

Figure 5: Posição Geográfica do Usuário. Fonte: Próprio Autor.

pessoa com deficiência visual possa habilitar uma pessoa de sua escolha para auxiliá-la em situações de emergência. Na rede social, utilizou-se a API (Application Programming Interface) do Google maps para mostrar a última posição geográfica do usuário.

\section{EXPERIMENTOS E RESULTADOS}

Experimentos foram realizados com o equipamento vestível, visando avaliar a eficácia do dispositivo em ambientes externos quanto ao aspecto comunicação entre os componentes e a rede.

O cenário de configuração dos experimentos foi organizado como segue: em uma rua com a presença de obstáculos reais, 2 pessoas 
sem deficiência visual utilizaram o equipamento vestível. Cada pessoa com o equipamento realizou um percurso de 650 metros (toda a extensão da rua). O percurso foi repetido por 3 vezes com cada indivíduo. O intervalo de confiança apresentado nos resultados é de $95 \%$.

Ressalta-se, que os experimentos realizados tem por objetivo avaliar o comportamento do dispositivo quanto a identificação correta dos obstáculos em ambientes externos. Nesta fase, não foram avaliadas a percepção/aceitação de pessoas com deficiência visual sobre o equipamento.

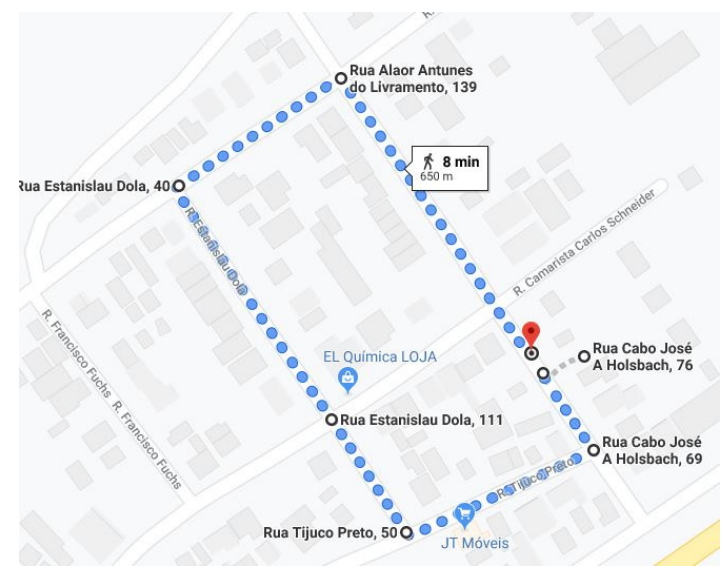

Figure 6: Mapa de Percurso dos Testes. Fonte: Próprio Autor.

Na Figura 6, apresenta-se o mapa do percurso realizado nos testes. O equipamento vestível foi conectado à Internet através do smartfone do usuário que está conectado a rede de telefonia celular. Contudo, para a conexão da pulseira com o equipamento vestível, o smartfone foi configurado como roteador e a tecnologia Wifi do padrão IEEE $802.11 \mathrm{n}$ foi utilizada para estabelecer a conexão e para a troca de mensagens entre os dispositivos.

A posição geográfica do utilizador é cadastrada na plataforma ThingSpeak a cada 1 minuto. Os dados da plataforma ThingSpeak são utilizados para apresentar o caminho percorrido pelo usuário.

No percurso, os utilizadores do dispositivo encontraram os seguintes obstáculos: 1 Telefone Público e 12 obstáculos do tipo Lixeira Basculante (Figura 7). Os obstáculos foram cadastrados antes da realização dos testes através do aplicativo Android (Figura 4), com o objetivo de verificar a assertividade do dispositivo quanto ao aviso dos obstáculos já cadastrados.

Na Tabela 3, é apresentado o comportamento do equipamento em relação a identificação de novos obstáculos. Estes testes foram realizados antes dos obstáculos estarem cadastrados no banco de dados. A identificação dos obstáculos com o sensor ultrassônico ocorreram a uma distância média de 1,23 metros. Na tabela, IC é o intervalo de confiança.

Observou-se que o equipamento fez a leitura de novos obstáculos com uma assertividade média de $87,35 \%$.

Na Tabela 4, apresenta-se os resultados obtidos nos testes quanto a leitura correta dos obstáculos cadastrados no banco de dados e o número de avisos emitidos para o utilizador do equipamento.

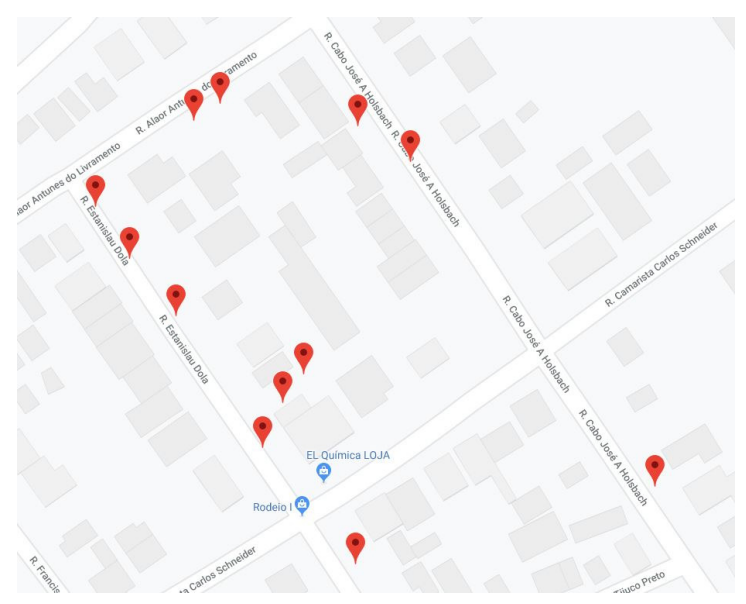

Figure 7: Obstáculos do Percurso. Fonte: Próprio Autor.

\begin{tabular}{|l|l|l|l|}
\hline Pessoa & $\begin{array}{l}\text { No Ob- } \\
\text { stáculos } \\
\text { Novos }\end{array}$ & $\begin{array}{l}\text { Leituras } \\
\text { Corretas }\end{array}$ & $\begin{array}{l}(\%) \text { Leituras Cor- } \\
\text { retas / IC }\end{array}$ \\
\hline P1 - 1 & 11 & 9 & $81,81 \% / 0,4382$ \\
\hline P1 - 2 & 10 & 8 & $80,0 \% / 0,4382$ \\
\hline P1 - 3 & 10 & 9 & $90,0 \% / 0,4382$ \\
\hline P2 - 1 & 13 & 12 & $92,3 \% / 0,4382$ \\
\hline P2 - 2 & 10 & 10 & $100,0 \% / 0,0$ \\
\hline P2 - 3 & 10 & 8 & $80,0 \% / 0,4382$ \\
\hline
\end{tabular}

Table 3: Resultados com o Equipamento Vestível - Obstáculos Novos. Fonte: Próprio Autor.

\begin{tabular}{|l|l|l|l|l|}
\hline Pessoa & $\begin{array}{l}\text { Leituras } \\
\text { Corretas }\end{array}$ & $\begin{array}{l}(\%) \\
\text { Leituras } \\
\text { Corre- } \\
\text { tas/IC }\end{array}$ & $\begin{array}{l}\text { Avisos } \\
\text { Antecipa- } \\
\text { dos }\end{array}$ & $\begin{array}{l}\text { (\%) Avisos } \\
\text { Antecipa- } \\
\text { dos/IC }\end{array}$ \\
\hline P1 - 1 & $10 / 12$ & $83,3 \% / 0,4$ & $11 / 12$ & $91,6 \% / 0,4$ \\
\hline P1 - 2 & $11 / 12$ & $91,6 \% / 0,4$ & $9 / 12$ & $75 \% / 0,4$ \\
\hline P1 - 3 & $11 / 12$ & $91,6 \% / 0,4$ & $11 / 12$ & $91,6 \% / 0,4$ \\
\hline P2 - 1 & $10 / 12$ & $83,3 \% / 0,4$ & $7 / 12$ & $58,3 \% / 0,4$ \\
\hline P2 - 2 & $12 / 12$ & $100 \% / 0,0$ & $10 / 12$ & $83,3 \% / 0,4$ \\
\hline P2 - 3 & $11 / 12$ & $91,6 \% / 0,4$ & $11 / 12$ & $91,6 \% / 0,4$ \\
\hline
\end{tabular}

Table 4: Resultados com o Equipamento Vestível - Obstáculos Cadastrados. Fonte: Próprio Autor.

O equipamento vestível apresentou um percentual médio de $90 \%$ em relação a leitura correta da posição dos obstáculos já cadastrados no banco de dados. No aspecto "Aviso Antecipado", ou seja, notificou antecipadamente o utilizador, o equipamento apresentou um percentual médio de acertos de $81,9 \%$.

\section{CONCLUSÃO E TRABALHOS FUTUROS}

Neste trabalho, foi apresentado o desenvolvimento e análise de desempenho de um equipamento vestível para auxiliar pessoas 
com deficiência visual quanto ao aspecto mobilidade, focado nas limitações da bengala convencional.

Conforme os resultados obtidos, verificou-se que o equipamento vestível avisa o usuário da existência de obstáculos já cadastrados como, lixeiras basculantes com um percentual de acertos acima de $81 \%$, e uma assertividade média de $87,35 \%$ na identificação de novos obstáculo.

Observou-se, que em alguns testes ocorreram atrasos na verificação de obstáculos cadastrados no banco de dados. Por exemplo, em um teste realizado com o usuário $P 2$ o equipamento avisou corretamente sobre 7 obstáculos dos 12 cadastrados, 58,3\%.

Como trabalhos futuro sugerem-se, uma melhoria do equipamento vestível para que o mesmo obtenha maior assertividade no anúncio de obstáculos novos e pré-cadastrados, e a realização de experimentos com pessoas com deficiência visual.

Outras melhorias possíveis são: a adição de um módulo que converte texto em voz, e o registro automático de obstáculos detectados durante o percurso do utilizador.

\section{REFERENCES}

[1] Arduiniana. 2020. Arduiniana. Disponível em: http://arduiniana.org/. Acesso em: 14 fev. 2020.

[2] Miguel Angelo Baggio, Gabriel Kist, Roberta Francine Schmachtenberg, Luciano Porto de Lima, Fábio Lorenzi da Silva, and Jean da Rolt Joaquim. 2015. PADEVI-Protótipo de Auxilio a Deficientes Visuais. Revista de Empreendedorismo, Inovação e Tecnologia 1, 1 (2015), 45-57.

[3] IBGE. 2010. Indicadores de desenvolvimento sustentável: Brasil 2010. IBGE, https://censo2010.ibge.gov.br/.

[4] Nishant Banat James and Ashish Harsola. 2015. Navigation aiding stick for the visually impaired. In 2015 International Conference on Green Computing and Internet of Things (ICGCIoT). IEEE, 1254-1257.

[5] Maria Marques, Atos Lima, and Marcelo Santos. 2016. Protótipo de Bengala Inteligente de Baixo Custo para o Auxílio de Deficientes Visuais. In Anais dos Workshops do Congresso Brasileiro de Informática na Educação, Vol. 5. 1344.

[6] Zeeshan Saquib, Vishakha Murari, and Suhas N Bhargav. 2017. BlinDar: An invisible eye for the blind people making life easy for the blind with Internet of Things (IoT). In 2017 2nd IEEE International Conference on Recent Trends in Electronics, Information \& Communication Technology (RTEICT). IEEE, 71-75.

[7] E SathyaNarayanan, BP Nithin, P Vidhyasagar, et al. 2016. IoT based smart walking cane for typhlotic with voice assistance. In 2016 Online International Conference on Green Engineering and Technologies (IC-GET). IEEE, 1-6.

[8] U-Blox. 2019. u-blox 6 GPS Modules Data Sheet. Disponível em: https://www.ublox.com. 